\section{Die Vision einer Vision ist die Leitfunktion}

\author{
Bericht zur Frühjahrstagung der Sektion \\ Wissenschafts- und Technikforschung der \\ Deutschen Gesellschaft für Soziologie: \\ „Die Bedeutung technischer Zukunftsvor- \\ stellungen für die Entwicklung, Einführung \\ und Bewertung neuer Technologien"
}

\section{Duisburg, 22.-23. Mai 2014}

\section{von Knud Böhle, ITAS}

Technische Zukunftsvorstellungen sind für die Entwicklung, Einführung und Bewertung neuer Technologien unbestreitbar von Bedeutung, so die Organisatoren der Veranstaltung Kornelia Konrad, Andreas Lösch, Martin Meister und Ingo Schulz-Schaeffer. Die Frage sei aber, wie sich die Wirkungen der Zukunftsvorstellungen analytisch erfassen und begründen lassen. Im Folgenden wird nach einigen allgemeinen Hinweisen zum Rahmen der Tagung ein Schnelldurchgang durch die einzelnen Beiträge unternommen, an den sich ein Resümee mit Überlegungen zu den Erträgen, dem Forschungsstand und -bedarf anschließt.

\section{Von Technikzukünften zur Technology Governance}

Etwa 40 Personen, Techniksoziologen, Wissenschafts- und Technikphilosophen, Politikwissenschaftler sowie Vertreter der Bereiche TA und Foresight, kamen im Kreißsaal eines inzwischen von der Universität Duisburg-Essen genutzten früheren Hospitals zusammen, um die 13 gebotenen Vorträge zu hören und zu diskutieren. ${ }^{1} \mathrm{Be}$ zogen auf die verhandelten Techniken überwogen Beiträge zu Energietechniken; bezogen auf die in bestimmten Formaten vorgefundenen Zukunftsvorstellungen, war am häufigsten von Szenarien und Visionen die Rede; bezogen auf die Themenstellung, dominierte die Frage nach der Bedeutung von Zukunftsvorstellungen im Kontext der ,Technology Governance“. Schon 2009 hatte übrigens Armin Grunwald in dieser Zeitschrift anlässlich der damaligen $4 \mathrm{~S}-$ Konferenz in Washington eine zunehmende Orientierung der STS-Community auf „Technology Governance“ festgestellt (Grunwald 2009). Eine STS-Forschung, die auf der
Ebene theoretischer Reflexion und empirischer Forschung dasselbe Feld bearbeitet wie die TA, ist für die TA-Community zweifelsohne relevant - und das gilt dann umstandslos auch für diese Tagung der Deutschen Gesellschaft für Soziologie.

\section{Themen und Thesen}

\subsection{Das Große-Ganze im Blick}

Alfred Nordmann, Wissenschaftsphilosoph an der TU Darmstadt, trug seine Kritik an der „Instrumentalisierung" von Zukunft eloquent und engagiert unter dem Titel „Design und Zeit“"vor, der an Heideggers „Sein und Zeit“ denken lassen soll. Da er die Zukunft grundsätzlich als der Vorstellung, dem Wissen, dem Planen und dem gestalterischen Zugriff grundsätzlich nicht zugänglich erachtet, richtet sich seine Kritik gegen Auffassungen von Zukunft, bei denen Zukunft als Objekt von Gestaltung und Kontrolle konzipiert wird, da sie in der Konsequenz dazu führten, dass Natur und Mensch gänzlich zum Gegenstand der Verbesserung und Leistungssteigerung würden. „Design“ betrachtet Nordmann als angemessene Übersetzung von „Gestell“, der Begriff, auf den Heidegger das totalisierende Wesen der modernen Technik brachte. Vor diesem Hintergrund kritisiert er die Hybris der Technowissenschaften, die Denkweise der „Visioneers" und „manche“ nicht näher genannte Ansätze der STS, TA und Technikethik. Die Anwesenden mochten den Vorwurf nicht auf sich beziehen und entgegneten, um einen wichtigen Einwand hier aufzugreifen, seine Wahrnehmung ihrer Praxis sei zu einseitig und unterschätze etwa den emanzipatorischen Gehalt normativer Szenarien, partizipativer Verfahren der Szenario-Erstellung oder von Realexperimenten.

Für Petra Schaper-Rinkel, Austrian Institute of Technology, steht außer Frage, dass Zukunftsszenarien de facto eine "politische Technologie“ oder anders gesagt Governance-Instrumente sind. Theoretisch ließen sie sich als „transdisziplinäre boundary objects" fassen, die als Bindeglied zwischen Stakeholdern aus Wirtschaft, Wissenschaft und Politikfeldern fungieren. In ihnen seien technische und soziale Innovationsprozesse in einen wechselseitigen Zusammenhang gebracht. Damit sie die intendierte Wirkung entfalten können, sei vorausgesetzt, dass die Perspektiven der 
unterschiedlichen Akteure, womöglich über partizipative Verfahren, Eingang in die Szenarien fänden. Auch wenn in Szenarien Zukunft zum Objekt werde, so könnten diese Objekte doch auch jederzeit dekonstruiert werden, indem etwa die eingesetzten Methoden, die Beteiligung oder auch Nicht-Beteiligung bestimmter Akteure beim Zustandekommen oder die politische Karriere bestimmter Szenarien (Bsp.: Bioökonomie) hinterfragt würden. Für weit schwieriger als solch eine Dekonstruktion, erachtet Schaper-Rinkel indes die Beantwortung der Frage, wie man die Zukunft als Praxis des Antizipierens untersuchen solle.

Für Armin Grunwald, ITAS, erbringen auf Prognosen oder Szenarien basierende Technikzukünfte selbstverständlich beratungsrelevante Orientierungsleistungen. Technikzukünften, die um die „new and emerging technologies“ gesponnen werden, und bei denen es gewissermaßen um fiktionale Bedeutungszuschreibungen geht, spricht er dagegen eine direkte Orientierungsleistung ab. Erst über eine hermeneutische Analyse dieser Technikzukünfte, die auf ihre Art auch Interventionen in laufende gesellschaftliche Zukunftsdebatten darstellten, ließe sich hier Orientierungswissen gewinnen. Die hermeneutische Analyse solcher Zukunftsvorstellungen könne über Bedeutungszuschreibungen, Werte und Interessen reflektieren und durchaus diagnostische Rückschlüsse auf die Gesellschaft ziehen, die diese Zukünfte hervorgebracht hat. In der Diskussion wurde darauf hingewiesen, dass in diesem Ansatz die wichtige Frage, welche Technikzukünfte im politischen Raum überhaupt zugelassen würden, nicht gestellt werde. Auch wurde ergänzt, dass es sich doch genauso lohnen könnte, die weniger spekulativen Technikzukünfte einer hermeneutischen Analyse zu unterziehen, denn auch sie sagten am Ende vermutlich mehr über die Gesellschaft aus, in der sie entstehen, als über die Zukunft.

\subsection{Energiewende und Energietechniken im Blick}

Stefan C. Aykut, Université Paris-Est Marne-laVallée, befasste sich mit den Funktionen von Szenarien in entscheidenden Phasen des Energiediskurses und der Energiepolitik der Bundesrepublik seit den 1950er Jahren. Szenarien wer- den dabei als komplexe, soziotechnische Objekte verstanden, die auf Modellrechnungen mit einer Vielzahl von makroökonomischen Variablen, Annahmen über technische Entwicklungen und politische Maßnahmen etc. basieren, die dann zu Aussagen über wahrscheinliche, wünschenswerte und/oder zu vermeidende Entwicklungen der Energienachfrage und des Energieangebots führen. Kommunizieren und Handeln in komplexen Verhältnissen könne vermutlich nicht ohne solche Wissensobjekte auskommen. Der historische Zugang, den Aykut gewählt hat, verdeutlichte zum einen den Wandel der angewandten Methoden von Prognose und Forecast zu Foresight und Szenarien und zum anderen die Abhängigkeit der Nutzung und der Wirkung der Szenarien von den je spezifischen Konstellationen von Wissenschaft, Öffentlichkeit und Politik in den einzelnen Phasen. Wichtig erscheint mir auch Aykuts Hinweis, dass Szenarien typischerweise im Plural vorkommen und unterschiedliche Zukünfte zulassen, ohne die Zukunft auf einen bestimmten Pfad festzulegen. Das ermögliche in der politischen Praxis Kompromisse und verhindere eine $\mathrm{zu}$ frühe Festlegung auf einen bestimmten Pfad.

Andreas Lösch und Christoph Schneider, beide ITAS, befassten sich mit Visionen als Bedingung der Möglichkeit von Realexperimenten, die neue soziotechnische Arrangements erproben. Realexperimente sollen Innovations- und Transformationsprozesse befördern und werden deshalb strategisch eingesetzt. Im untersuchten Fall ist die Überführung des gegenwärtig stark zentralisierten Energieregimes in neue soziotechnische Arrangements, die die Integration erneuerbarer Energien leisten können, das Ziel. Visionen, das wurde am Beispiel der Smart Grid-Vision verdeutlicht, seien eine Voraussetzung, alte und neue Akteure, sowie alte und neue technische Elemente zu verknüpfen, um damit im Prozess noch nicht Dagewesenes auf der Ebene von Wissen, Akteurspositionen und Governanceformen zu erzeugen und erfahrbar zu machen. Je nach Akteursposition variierten die Erkenntnisse und Erfahrungen natürlich stark. Das leuchtet ein. Die Frage, die sich aufdrängt ist allerdings, ob nicht zwei epistemische Ebenen, die für das Realexperiment Bedeutung haben, genauer zu unterscheiden wären: zum einen die Zukunftsvision als Bezugspunkt 
und zum anderen so etwas wie das Pflichtenheft oder die Versuchsanordnung des in der Gegenwart durchzuführenden Realexperiments.

Alexander Wentland, Technische Universität Berlin, befasste sich mit den auf Elektromobilität bezogenen soziotechnischen Imaginationen. Elektromobilität sei ein interessanter Fall, weil verschiedene Zukunftsbilder konkurrierten. Die Bandbreite der möglichen Innovationen sei groß. „Vehicle-to-Grid“ bezeichnet dabei eine besonders weitgehende Idee, weil Elektrofahrzeuge nicht nur als Substitut für Fahrzeuge mit Verbrennungsmotor gedacht werden, sondern auch als kleine, mobile und vernetzte Energiespeicher. Er konnte an diesem Beispiel zeigen, dass größere Narrative bedeutsam werden, wenn es um ,systemische“ Technik geht. Vorstellungen über die Zukunft von Urbanität, Umwelt und Lebensstilen sind dann mitentscheidend dafür, welche technologischen Entwicklungspfade konkret beschritten werden. Die Herangehensweise steht durchaus in der Traditionslinie der Technikgeneseforschung, aber die Blickrichtung hat sich verändert. Vom Blick auf die Genese von Artefakten wurde auf die Konstitution soziotechnischer Felder umgestellt.

Dirk Scheer, Universität Stuttgart, stellte ein sehr komplexes Forschungsprojekt zur Wirkung von Simulationswissen bei politischen Entscheidungsprozessen vor - konkretisiert und empirisch untersucht am Fallbeispiel „Carbon Capture and Storage“, das in Debatten zur Energie- und Klimapolitik eine große Rolle spielt. Untersucht wurden die Erkenntnis- und Kommunikationsfunktionen von Simulationen an der Schnittstelle von Wissenschaft und Politik und die Bedingungen der Politikrelevanz von Simulationen. Das meint konkret Perzeptions- und Rezeptionsmuster sowie Bewertungs- und Verwertungsmuster von Simulationswissen bei politisch-gesellschaftlichen Entscheidungsträgern. In der Bewertungsdimension wurde zwischen einer Instrumenten-, Prozess- und Ergebnisbewertung unterschieden, in der Verwertungsdimension zwischen instrumentellem, konzeptionellem, strategischem und prozeduralem Gebrauch von Simulationen in politischen Entscheidungsprozessen. Die Analytik, die Scheer hier exemplarisch für die Computersimulation erarbeitet hat, dürfte analog bei Szenarien und Modellen greifen, die gleichfalls zunächst Instrumente der Erarbeitung von Zukunftswissen sind, um dann als ,materialisierte“ Zukunftsvorstellungen (wie es auf einer Folie hieß) weiter wirksam zu werden.

\subsection{Die Informations- und Kommunikations- technologien im Blick}

Sascha Dickel, Technische Universität München, und Jan-Felix Schrape, Universität Stuttgart, erörterten „Spekulationsspiele“, also ein SichEinlassen auf utopische Leitbilder im Kontext soziotechnischer Transformationsprozesse. Das Erstaunliche dabei sei, dass Wissenschaftler sich auf die „Spekulationsspiele“ einließen, obwohl sie wissen dürften, dass die Ankündigungen radikaler Veränderungen später enttäuscht werden. An den Beispielen Web 2.0 und 3D-Druck wurde erläutert, wie diese etwa über Schlagworte wie ,prosumerism“ und „do-it-yourself" in einen gedanklichen Zusammenhang mit sozialphilosophischen Vorstellungen gebracht werden, die die fortgeschrittene kapitalistische Arbeitsteilung kritisieren. Sozialwissenschaftler sollten sich hüten, ihre Rolle darin zu sehen, zirkulierende Visionen mit sozialtheoretischen Generalutopien zu unterfüttern. Ein guter Punkt. Sinnvoller wäre es, in solchen Fällen zu analysieren, wie es zu unseriösen utopischen Konstruktionen kommt. Drei typische Fehleinschätzungen, die utopische Leitbilder begünstigen, wurden benannt: häufig würden Einzelfälle vorschnell generalisiert, die Bedeutung von „Visioneers“ und „,early adopters“ werde überschätzt und geschichtliche Erfahrungen und potenzielle Verlierer der Entwicklung würden ausgeblendet. Wichtiger noch als sich mit den Utopien zu befassen, könnte es aber sein, das gesellschaftlich Virulente der neuen Praxisformen genauer zu bestimmen.

Martin Meister und Nils Kubischok (weitere Autoren: Anna Herwig und Ingo Schulz-Schaeffer), Universität Duisburg-Essen, trugen Zwischenergebnisse aus dem laufenden DFG-Projekt „Szenarien als kognitive Orientierungsmuster in Technikgenese und Technikgestaltung (SKOTT)“ vor, in dem es um die Wirkungen und die Wirksamkeit von Zukunftsvorstellungen in den Technologiefeldern Ubiquitous Computing und $\mathrm{Na}$ notechnologie geht. Die Studie ist empirisch und international vergleichend (EU, USA, Japan) 
angelegt. Der Vortrag konzentrierte sich auf das Technologiefeld Ubiquitous Computing, das von Beginn an mit einer Vision und ausformulierten Szenarien (Mark Weiser) einherging. Szenarien im hier verwendeten Begriffssinn sind Darstellungen, die beschreiben, wie die mögliche zukünftige Wirklichkeit solcher Visionen konkret aussehen könnte. Szenarien sind folglich auf einer anderen Konkretionsstufe als Visionen angesiedelt und würden, so die zu überprüfende These, die Technikentwicklung orientieren. In Szenarien werde eine technische Lösung typischerweise als Problemlösung für einen bestimmten Fall in einem bestimmten Einsatzgebiet imaginiert (Bsp. Museumsführer). Solche Szenarien seien nachweislich in der Lage, Forschungsprozesse zu strukturieren und Forschungen zu koordinieren.

Es ist nicht ganz klar geworden, wie konkret die Szenarien waren bzw. in welchem Ausmaß reale Anforderungen bestimmter Anwendungsfelder darin berücksichtigt wurden. Wäre es so, dass sich Ingenieure mögliche Anwendungsszenarien bloß grob ausmalen müssten, um ihre weiteren Forschungen zu orientieren, wäre das ein erstaunlicher Befund. Wäre es dagegen so, dass die in Frage stehenden Szenarien als Anwendungsszenarien zu verstehen wären, also Leistungsanforderungen oder Pflichtenheften ähnelten, wäre das Szenario vor allem ein Instrument, um Erwartungen verschiedener Akteure zu kommunizieren und abzugleichen. In beiden Fällen aber scheint ein Szenario für die kognitive Orientierung der Forscher auszureichen. Es würde sich lohnen, den Szenariobegriff weiter zu klären, um dann die unterschiedlichen Funktionen, die Szenarien in Forschung- und Entwicklung einerseits und der Technology Governance andererseits erfüllen, besser unterscheiden zu können.

\subsection{Miszellen}

Katrin Hahn, TU Dortmund, thematisierte die Rolle komplementärer Erwartungen bei industriellen Innovationsprojekten (Bsp. Kooperation eines KMU mit einer Forschungseinrichtung zur Fertigung eines neuartigen Aluminiumzylinders), wobei es insbesondere um interpersonale Erwartungen ging, die für Abstimmungsprozesse, das Abstecken des Handlungsrahmen und die Struk- turierung des Innovationsprozesses wichtig seien. Die Beschäftigung mit diesem Innovationsprojekt - mit seinem vergleichsweise nahen Zukunftshorizont - mag im Kontext weitausgreifender Visionen zunächst etwas off topic erscheinen, aber das Gegenteil ist der Fall, weil eine zentrale Frage deutlich wird: Wenn alle Erwartungen Zukunftserwartungen und alle auf Neues bezogenen Vorstellungen Zukunftsvorstellungen sind, reicht dann nicht eine Analytik für Innovationsprozesse trotz extrem unterschiedlicher Zeithorizonte?

Clemens Blümel, Humboldt-Universität zu Berlin, ging von der gut nachvollziehbaren Annahme aus, dass neu entstehende Forschungsfelder, hier konkret die Synthetische Biologie, sich gegenüber Akteuren in Politik und Gesellschaft in besonderer Weise rechtfertigen müssen, um sich langfristig zu etablieren. Die weitere Annahme war dann, dass auch im wissenschaftlichen Raum, genauer im Bereich des wissenschaftlichen Publizierens zunehmend gesellschaftliche Erwartungen adressiert würden. Untersucht wurde das Genre „Übersichtsartikel“ (Review) mit dem Ergebnis, dass in der Tat außerwissenschaftliche Erwartungen in das wissenschaftliche, begutachtete Publikationswesen Einzug halten und dieses transformieren. Das ist ein wichtiger Befund. Die interessante Frage, ob diese Entwicklung bei anderen Technowissenschaften eher stärker oder schwächer ausgeprägt ist, konnte nicht geklärt werden.

Frank Heidmann und Gregor Glass (Mitautor: Anouk Meissner), FH Potsdam, hatten ihren beispiel- und bildreichen Vortrag wortreich „Webbasierte Visualisierung und Exploration von Bildern der Mensch-Technik-Kooperation als Toolbox für Zukunftsforschung, Innovationsmanagement und Technikethik" überschrieben. Die Beispiele, die sie gaben, umfassten u. a. so unterschiedliches wie die Visualisierung von BigData-Mengen in Echtzeit, eine „multi-user“fähige, interaktive Verkehrssimulation auf einem Bildschirmtisch (tabletop) als Input für Gruppendiskussionen und eine Datenbank, in der ScienceFiction-Filme abgespeichert und hinsichtlich der in ihnen dargestellten Mensch-Computer-Interaktionen ausgewertet werden können. Es wurde deutlich, dass Informationsdesigner der STS-Forschung und der TA etwas zu bieten haben. 


\section{Resümee und Ausblick}

Die Tagung hatte versprochen, die Frage zu behandeln, wie sich die Wirkungen von Zukunftsvorstellungen analytisch fassen und begründen lassen. Die Frage nach dem wie, wurde in der Regel funktionalistisch beantwortet: Zukunftsvorstellungen als boundary objects, als Bedingung der Möglichkeit bestimmter Kommunikations-, Kooperations- und Entscheidungsprozesse, als conditio-sine-qua-non der Governance von Innovations- und Transformationsprozessen. Das wurde auch in den Fallbeispielen sehr deutlich, die sich auf kommunikativ und medial materialisierte Zukunftsvorstellungen, etwa Szenarien oder Computersimulationen, konzentrierten. Zukunft wird in diesen Formen zwar zum Wissensobjekt, aber es geht dabei um Surrogate: provisorische, nur temporär nützliche Objekte, die durch ihren Charakter letztlich bestätigen, dass man über die Zukunft nicht verfügen kann.

Auf der Tagung zeichneten sich auch Konturen eines kritischen und selbstkritischen Umgangs mit diesen Wissensobjekten ab. Die Frage nach den im politischen Raum nicht zugelassenen Zukünften gehörte dazu, ebenso die Frage, in wieweit Szenarien und dergleichen strategisch eingesetzt und inszeniert werden. Dazu gehörte auch der Hinweis auf die Spekulationsspiele und „gehypten“ Zukünfte, auf die sich Sozialwissenschaftler (gegen besseres Wissen) einlassen und die sie in manchen Fällen selbst unkritisch bedienen. Auch die Analyse von soziotechnischen Zukunftsvorstellungen, um freizulegen, was sie an Gegenwartsdiagnose enthalten, ein Verfahren das bei der Interpretation von literarischen Utopien gang und gäbe ist, erlaubt Kritik. Hilfreich war in dem Zusammenhang auch der Hinweis von philosophischer Seite, den unhintergehbaren Gegenwartsbezug all dieser Zukünfte nicht aus den Augen zu verlieren. Ein sparsamerer Gebrauch des Wortes „Zukunft“ erscheint angeraten: ein Szenario muss nicht Zukunftsszenario heißen und ein Gedankenexperiment nicht gleich Zukunftsvision. Das Wissen um die Immanenz der Gegenwart enthebt andererseits aber nicht der Aufgabe, a) die Eigenzeiten unterschiedlicher Prozesse in Rechnung zu stellen (von z. B. Legislaturperioden, Haltbarkeitszeiten von Produkten, Infrastrukturwandel) und b) die unterschiedlichen Formen der Zeitbewirtschaftung in Szenarien, Roadmaps,
Narrativen, Visionen, Leitbildern etc. in der ausgedehnten Gegenwart differenziert zu betrachten.

Auf der konzeptionellen Ebene würde ich weiteren Forschungsbedarf auch bei den häufig gebrauchten Termini „Erwartung“ und „Orientierung" sehen. Soziologisch bleibt doch häufig zu unklar, wer da eigentlich was von wem auf welcher Grundlage erwartet. Fraglich ist auch, wie man Orientierungswissen untersuchen kann, das ja nicht nur kognitiven Ansprüchen eines besseren Wissens genügen muss, sondern streng genommen nur dann gegeben ist, wenn es nachweislich eine Orientierungsleistung für jemanden erbringt. $\mathrm{Zu}$ wenig soziologisch wird möglicherweise auch noch die Binnenstruktur der Zukünfte untersucht, die nicht epistemisch, sondern sozioepistemisch aufzufassen ist. Ein Leitbild, um das hier mit Hellige (1996) zu erläutern, ist ja nicht einfach ein Leitbild, sondern ein „Bündel technischer Kriterien, soziotechnischer Zielsetzungen, kultureller Bedeutungszuweisungen, organisatorischer Strukturbedingungen und gesellschaftlicher Nutzungs- bzw. Wirkungserwartungen“. Ähnliches ließe sich von Visionen sagen.

Schließlich wäre es für die Weiterentwicklung der Analytik von Zukünften hilfreich, die Unterscheidung von Leitvorstellungen und Visionen beizubehalten. Von einer Leitvorstellung (Leitprinzip, Leitbild etc.) wäre dann zu sprechen, wenn eine konkrete gegenwärtige, im Einzelnen durchaus neuartige Praxis (Technikentwicklung, Realexperiment, Modellversuch, Förderprogramm) angeleitet, instruiert und strukturiert wird, während Visionen im Hypothetischen ,wenn, dann...“ anzusiedeln sind und ihre realen Wirkungen auf gegenwärtige Diskurse und Handlungen über den gedanklichen Vorlauf (Gedankenexperimente) ausüben. Beide Formen stehen in Beziehung: Leitvorstellungen werden oft visionäre Ideen integrieren und Visionen werden versuchen, wie im Titel des vorliegenden Tagungsberichts behauptet, sich hin zu Leitvorstellungen zu entwickeln.

\section{Anmerkung}

1) Das Tagungsprogramm findet sich online unter: http://www.soziologie.de/fileadmin/user_upload/ Sektion_WissenschaftundTechnik/TagungsprogrammZukunftsvorstellungenDuisburg2014.pdf (download 8.10.14). 


\section{Literatur}

Grunwald, A., 2009: STS und TA - Programmatische Annäherungen? Bericht vom 34. Annual Meeting der Society for the Social Study of Science. In: Technikfolgenabschätzung - Theorie und Praxis 18/3 (2009), S. $105-108$

Hellige, H.D., 1996: Technikleitbilder als Analyse-, Bewertungs- und Steuerungsinstrumente: Eine Bestandsaufnahme aus informatik- und computerhistorischer Sicht. In: Hellige, H.D. (Hg.), Technikleitbilder auf dem Prüfstand. Leitbild-Assessment aus Sicht der Informatik- und Computergeschichte. Berlin, S. 15-35

$《 》$

\section{Sustainable Cities: Challenges and Opportunities at Different Scales}

Report from the International Conference "Sustainability 2014: Future Urban Development at Different Scales"

Karlsruhe, Germany, May 7-9, 2014

\section{by Alexandra Quint and Marius Albiez, ITAS}

In a phase of (re-)urbanization of societies, cities all over the world face the challenge to reinvent themselves in line with sustainable development (cf. Fücks 2011). Climate change, limited resources, demographic developments, debt crisis, deterioration of the ecological sphere, social inequality and other issues urgently call for action in terms of urban development. On the one hand, cities are spaces where the mentioned challenges accumulate as cities are their causers. On the other hand, due to the density of innovation, creativity and knowledge inherent to urban spaces, they are an important factor when it comes to facing the challenge of sustainability transformation.

TA research has been dealing with sustainable development for years now. At ITAS, there is a focus on sustainability and ecology research especially in the fields of energy systems, land use and resources. In particular the research activities in the field of sustainable urban development were intensified at the institute in the last years. ${ }^{1}$ Therefore it is hardly surprising that the international research initiative "Forum on Sustainable Technological
Development in a Globalizing World", of which ITAS has been a member since its foundation in 2002, held its annual international conference with the title "Sustainability 2014: Future Urban Development at Different Scales" in Karlsruhe. The conference brought together experiences from theory and practice as well as different cultural and disciplinary perspectives on current issues of sustainable urban development at different scales and in different local contexts. Main aspects of the discussions will be outlined here.

\section{Designing the Existing}

Especially the existing cities of the Western world pose a challenge for sustainable development. The new construction rate in the German housing sector was below 1 percent for years (cf. Rexroth et al. 2014, p. 18). Current and future demographic developments which will make Western societies shrink make it clear that dealing with the existing urban environments is of prime importance for a sustainable urban development. How can already existing urban spaces - neighbourhoods, district, cities and urban regions - be designed and transformed to be more sustainable?

The presentation of Freya Brandl focused on communal living in Vienna in the context of ageing Western societies. In Vienna, more and more elder singles live on their own - with consequences for the well-being of the residents. The concept of the project "eco-com.60+" is to convert existing multi-family houses into communal apartments where single kitchens or living rooms are merged into one communal room. Separate apartments for the privacy of the residents remain. The aim is to increase the number of apartments in an overstretched housing market, foster energy efficiency as well as social exchange and responsibility within the neighbourhood.

The overall objective of the "District Future - Urban Lab" project is to transform an existing district of the city of Karlsruhe (Germany) into a sustainable one. How is this to be done? In his presentation, Marius Albiez described the transdisciplinary approach of the research project at the Karlsruhe Institute of Technology. The project intends to bring together knowledge and technological innovation of the local university with 